\title{
Catheter Ablation of Ventricular Tachycardia in LMNA Cardiomyopathy: Out of Sight but not Out of Mind
}

\author{
Ahmad Halawa ${ }^{1}$, Paul Zei ${ }^{2}$, Neal Lakdawala ${ }^{1}$, William Sauer ${ }^{1}$, Usha Tedrow ${ }^{2}$, and Sunil \\ Kapur $^{2}$ \\ ${ }^{1}$ Brigham and Women's Hospital Carl J and Ruth Shapiro Cardiovascular Center \\ ${ }^{2}$ Brigham and Women's Hospital
}

July 12, 2021

\begin{abstract}
Lamin Cardiomyopathy (LC) is associated with refractory ventricular arrhythmias. Catheter ablation success rate is low due to presence of multiple circuits and intramural substrate. We present a LC case presented with electrical storm. During catheter ablation, arrhythmia was easily inducible but activation mapping, including full epicardial and endocardial mapping, failed to demonstrate the full tachycardia cycle length ( $70 \%$ only) suggesting intramural activation. Critical isthmus was not identified even with successful concealed entrainment on both Endo/epicardial surfaces. This case shows that even combined endocardial and epicardial catheter approach can be ineffective in identifying the full arrhythmogenic substrate in LC.
\end{abstract}

\section{Introduction:}

The dominant mutations in LMNA patients, a gene which encodes the nuclear lamina proteins Lamin A and $\mathrm{C}$, account for ${ }^{\sim} 5 \%^{1}$ of dilated cardiomyopathy. The penetrance of LMNA mutations is age-dependent and associated with high rates of AV block, atrial and ventricular arrhythmias, and ultimately progressive systolic dysfunction with high rates of end stage heart failure.

Early cardiac clinical manifestations include first degree AV block with evident imaging correlates of AV nodal and ventricular septal fibrosis. ${ }^{2}$ Ventricular arrhythmias (VA) in patients with LMNA cardiomyopathy are malignant, often refractory to medical management and requires iterative device programming and frequently catheter ablations $(\mathrm{CA})^{3,4}$

Advanced catheter ablation techniques including radiofrequency ablation (RF), surgical cryoablation and epicardial mapping/ablation may offer palliation of recurrent VA, but the prognosis remains poor. Both recurrent VA and progressive heart failure are common in patients even after catheter ablation. ${ }^{5}$ Challenging intramural and deep septal scar substrates have been described as reason for catheter ablation failure in nonischemic cardiomyopathy patients even with the lack of fibrosis on imaging studies. ${ }^{6}$ To further demonstrate the challenge of managing VA in patients with LMNA cardiomyopathy, we present a case of refractory ventricular arrhythmia to medical and catheter ablation therapies.

\section{Case Report:}

A 50-year-old male with LMNA cardiomyopathy and NYHA class III and left ventricular ejection fraction (LVEF) of $35 \%$ was admitted with electrical storm. His initial presentation was atrial fibrillation in 2007, left bundle branch block and cardia arrest in 2017, following which cardiomyopathy diagnosed and extensive work up including genetic testing revealed that he was haplo-insufficient for LMNA due to a microdeletion of chromosome $1 .{ }^{7}$ He underwent placement of a secondary prevention defibrillator/cardiac re-synchronization 
therapy (CRT-D) following cardiac arrest and recovery. Despite optimal medical therapy and resynchronization therapy, his LVEF continued to decline, and he developed frequent episodes of ventricular tachycardia (VT). One month before admission, he underwent catheter ablation with substrate modification at the periaortic area of the left ventricle but continued to have uncontrolled arrhythmia. At that time, Computed tomography of the heart did not suggest myocardial replacement fibrosis, but the images were limited by intracardiac device artifact.

After catheter ablation, arrhythmia was managed with Amiodarone 400mg a day and Metoprolol 200mg a day but continued to have high burden of self-limited but symptomatic slow VT. Three weeks later, he suffered frequent prolonged episodes of slow VT with CL of $460 \mathrm{~ms}$ below the rate of detection in the device's slowest therapy zone $(420 \mathrm{~ms})$. Beside palpitations, he was hemodynamically stable and presented to outpatient clinic. After immediate transferred to the emergency room, a successful manual anti-tachycardia pacing (ATP) converted his rhythm to sinus rhythm. He was referred to the electrophysiology laboratory for mapping and CA of electrical storm.

Procedure description:

The procedure was performed under conscious sedation then changed to general anesthesia during epicardial mapping and ablation. Two catheters were used for the purpose of mapping and ablating: multipolar mapping catheter (PentaRay ${ }^{\circledR}$ ) and 3.5-mm tip irrigated catheter (Thermocool SMART-TOUCH; Biosense Webster). Endocardial/epicardial voltage maps were created by using a three-dimensional (3D) electroanatomic mapping system (CARTO, Biosense Webster). Bipolar electrogram amplitude of $<1.5 \mathrm{mV}$ was defined as low- voltage zone consistent with scar. Dense scar was defined as voltage [?]0.5 mV. Images integration with the preprocedural contrast-enhanced computer tomography scans and the 3D mapping system images were used to identify and reconstruct LV and coronary arteries anatomy. Catheter ablation sites were chosen based on entrainment, activation, and substrate mapping.

Initially, trans-septal access was utilized to map the left ventricle endocardium then changed to retrograde arterial access for further reach of targeted substrates. During endocardial mapping, extensive low-voltage substrates were noted at the peri-aortic area with extension to the sub-aortic/mitral continuity and the peri-mitral valve areas, with normal endocardial bipolar voltage in the remainder.

Programmed ventricular stimulation from the RV catheter with stimulation at 600/320 millisecond (ms) induced the clinical VT. We were able to induce four VT morphologies during the procedure (Figure 1). Entrainment from the endocardial space at the periaortic area showed concealment and short stimulation to QRS indicating VT exit site. Despite successful concealed entrainment at the peri-aortic area, complete tachycardia CL mapping was not possible during endocardial approach (Figure 2). Earliest sites of activation during clinical VT were noted to be a wide area at the anteroseptal wall. See figure 3. Radiofrequency (RF) ablation lesions were performed at this site. Extensive RF ablation was performed by targeting all areas of fractionated, late potentials and long stimulus to QRS sites. In addition, we ablated at the septal aspect of the right ventricle outflow tract (RVOT) opposing the left ventricle outflow tract (LVOT) to bracket the targeted area.

Simultaneous endocardial unipolar voltage mapping indicated possible extensive epicardial scar. See figure 2. After anticoagulation reversal, epicardial access was obtained using the Needle-in-needle technique. ${ }^{8}$ A Decapolar catheter (Webster ${ }^{(r)}$ Decanav) was inserted through a long 8 French sheath to the epicardial space and used for mapping and pacing purposes. Low voltage areas were noted anterior to the LVOT with anterior extension toward the apex and at the basal portion of the lateral wall (Figure 2). Ventricular sites with fractionation and late potentials were marked during voltage mapping at the epicardium. Pace mapping was performed to evaluate the best match with the clinical VT. Coronary artery territory and areas of phrenic capture were marked. Clinical VT was induced by pacing from the RV catheter. Despite extensive epicardial activation mapping during VT, the full CL was not completely mapped. (Figure 3). Best entrainment from the epicardium was at the LV anterior wall with concealed fusion and long postpacing interval indicating bystander site. Multiple RF lesions were delivered at the epicardial space that 
demonstrated good entrainment properties, pace mapping closer to the morphology of clinical VT, earliest pre-systolic site or sites with the long stem to QRS period. During ablation we utilized RF power of 50 watts, and half normal saline to produce deeper lesions at presumed intra-mural substrate in locations deemed to be safe and $>10 \mathrm{~mm}$ apart from the marked coronary arteries. ${ }^{9}$ Thereafter, attempts to re-induce clinical VT have failed and the target VT was rendered non-inducible. The patient was hemodynamically stable after the procedure and was transferred back to the cardiology floor. After 48 hours of observation, without significant arrhythmia, he was discharged home on Amiodarone. He was hospitalized one month later with frequent ATP and required quinidine treatment which was effective. Both Amiodarone and Quinidine were slowly weaned off later because of side effects. Six months since ablation, he has been free of any significant ventricular arrhythmia or ICD shocks. Because of worsening cardiac function, he is currently undergoing thorough evaluation for cardiac transplantation.

\section{Discussion:}

This case presentation reflects the complex nature of VT in patients with LMNA cardiomyopathy. Despite thorough mapping, the full VT circuit was not detected, which was attributed to a hidden mid-myocardial isthmus not detected during activation mapping on opposite surfaces. Previous case series have observed similar findings of extensive intramural substrate in similar patients. ${ }^{10}$

Mid myocardial substrate dependent arrhythmias are difficult to define but can be suggested by threedimensional circuit activation with missing complete reentrant patterns during simultaneous endo/epicardial mapping. ${ }^{11}$ Other features suggesting intramural ventricular arrhythmias include intramural fibrosis on imaging with CT or MRI. Large areas of earliest activation, during activation mapping, suggest the critical portion of the circuit is not being mapped. Additionally, RF application at the site of earliest activation without termination of VT suggests intramural ventricular arrhythmia. Intramural substrate is not limited to LMNA patients, and has been seen in 44-61\% of ischemic and non-ischemic ventricular arrhythmia patients. ${ }^{10,11}$

The morphology variability encountered in our patient likely reflects the complexity of the intramural reentry circuit with variable cycle lengths and exits with inaccessible substrate in each mapped VT morphology. ${ }^{11}$

This phenomenon likely explains the variable VT morphologies and cycle lengths in this patient. ${ }^{12}$ Not unexpectedly, clinically there is a higher incidence of catheter ablation failure, arrhythmia recurrence and poor prognosis of VT in patients with LMNA cardiomyopathy due to complex intramural substrate. ${ }^{4,5}$

Complete substrate elimination may not be effective or possible with standard ablation approaches in intramyocardial substrate cases. All attempts to map the arrhythmia may be limited to the encountered inner/outer loops and multiple entrance and exit sites. ${ }^{13}$

Relying on traditional catheter ablation techniques is often insufficient and carries higher risk of recurrence. Careful coordination between electro-anatomical mapping, VT entrainment results and pre-procedure imaging studies is recommended when possible. Utilizing nonstandard techniques, including half normal saline radiofrequency ablation, bipolar ablation, simultaneous unipolar catheter ablation, or needle catheter ablation could be critical to eliminate deep substrate components and essential for successful ablation. ${ }^{9}$ Unfortunately, even with the best efforts, recurrent ventricular tachycardia is associated with adverse events in LMNA cardiomyopathy and other advanced heart failure therapies should be considered early for these patients once VT emerges. ${ }^{5}$

\section{Conclusion:}

Malignant ventricular arrhythmias are a challenge in the management of LMNA cardiomyopathy patients. The probability of midmyocardial substrate is high in these patients and sometimes the use of an endocardial and epicardial catheter approach is not effective in identifying arrhythmogenic substrate. Careful planning in a LMNA-experienced center with the availability of ablation tools to target the mid-myocardium may optimize chances of successful catheter ablation. 


\section{References:}

1. Hasselberg NE, Haland TF, Saberniak J, Brekke PH, Berge KE, Leren TP, Edvardsen T, Haugaa KH: Lamin A/C cardiomyopathy: young onset, high penetrance, and frequent need for heart transplantation. Eur Heart J [Internet] 2018; 39:853-860. Available from: http://www.ncbi.nlm.nih.gov/pubmed/29095976

2. Arbustini E, Pilotto A, Repetto A, Grasso M, Negri A, Diegoli M, Campana C, Scelsi L, Baldini E, Gavazzi A, Tavazzi L: Autosomal dominant dilated cardiomyopathy with atrioventricular block: a lamin A/C defect-related disease. J Am Coll Cardiol [Internet] 2002; 39:981-990. Available from: https://linkinghub.elsevier.com/retrieve/pii/S0735109702017242

3. van Rijsingen IAW, Arbustini E, Elliott PM, et al.: Risk Factors for Malignant Ventricular Arrhythmias in Lamin A/C Mutation Carriers. J Am Coll Cardiol [Internet] 2012; 59:493-500. Available from: https://linkinghub.elsevier.com/retrieve/pii/S0735109711048443

4. Sidhu K, Han L, Picard KCI, Tedrow UB, Lakdawala NK: Ventricular tachycardia in cardiolaminopathy: Characteristics and considerations for device programming. Hear Rhythm [Internet] 2020; 17:1704-1710. Available from: https://linkinghub.elsevier.com/retrieve/pii/S1547527120304574

5. Kumar S, Androulakis AFA, Sellal J-M, et al.: Multicenter Experience With Catheter Ablation for Ventricular Tachycardia in Lamin A/C Cardiomyopathy. Circ Arrhythmia Electrophysiol [Internet] 2016; 9. Available from: https://www.ahajournals.org/doi/10.1161/CIRCEP.116.004357

6. Liang JJ, D'Souza BA, Betensky BP, et al.: Importance of the Interventricular Septum as Part of the Ventricular Tachycardia Substrate in Nonischemic Cardiomyopathy. JACC Clin Electrophysiol [Internet] 2018; 4:1155-1162. Available from: https://linkinghub.elsevier.com/retrieve/pii/S2405500X18303840

7. Cirino AL, Cuddy S, Lakdawala NK: Deletion of entire LMNA gene as a cause of cardiomyopathy. Hear case reports [Internet] 2020; 6:395-397. Available from: http://www.ncbi.nlm.nih.gov/pubmed/32695585

8. Kumar S, Bazaz R, Barbhaiya CR, et al.: "Needle-in-needle" epicardial access: Preliminary observations with a modified technique for facilitating epicardial interventional procedures. Hear Rhythm [Internet] 2015; 12:1691-1697. Available from: http://www.ncbi.nlm.nih.gov/pubmed/2588599

9. Tzou WS, Rothstein PA, Cowherd M, et al.: Repeat ablation of refractory ventricular arrhythmias in patients with nonischemic cardiomyopathy: Impact of midmyocardial substrate and role of adjunctive ablation techniques. J Cardiovasc Electrophysiol [Internet] 2018; 29:1403-1412. Available from: http://doi.wiley.com/10.1111/jce.13663

10. Bhaskaran A, Nayyar S, Porta-Sanchez A, Jons C, Masse S, Magtibay K, Aukhojee P, Ha A, Bokhari M, Tung R, Downar E, Nanthakumar K: Direct and indirect mapping of intramural space in ventricular tachycardia. Hear Rhythm 2020; 17:439-446.

11. Tung R, Raiman M, Liao H, et al.: Simultaneous Endocardial and Epicardial Delineation of 3D Reentrant Ventricular Tachycardia. J Am Coll Cardiol 2020; 75:884-897.

12. Martin R, Hocini M, Haisaguerre M, Jais P, Sacher F: Ventricular Tachycardia Isthmus Characteristics: Insights from High-density Mapping. Arrhythmia Electrophysiol Rev [Internet] 2019; 8:54-59. Available from: https://www.aerjournal.com/articles/ventricular-tachycardia-isthmus

13. Gizurarson S, Spears D, Sivagangabalan G, Farid T, Ha ACT, Masse S, Kusha M, Chauhan VS, Nair K, Harris L, Downar E, Nanthakumar K: Bipolar ablation for deep intra-myocardial circuits: human ex vivo development and in vivo experience. Europace [Internet] 2014; 16:1684-1688. Available from: http://www.ncbi.nlm.nih.gov/pubmed/24554525

\section{Figure legends:}

Figure 1: Surface electrocardiograms of different ventricular tachycardias (VT) induced during the electrophysiology study. The small variation in morphologies and cycle lengths, indicate variable exits of intramural 
substrates. Milliseconds: ms.

Figure 2: $\mathrm{A}$ and $\mathrm{B}$ are showing endocardial voltage mapping with peri-aortic and peri-mitral annulus low voltage areas indicating scar.

$\mathrm{C}$ and D showing RAO and LAO projections of the epicardial voltage mapping. Low voltage zones are noted at the antero-septal wall and the basal anterolateral wall.

Figure 3: Endocardial and epicardial coherent activation mapping during ventricular tachycardia. A and B figures show complex reentry circuit in the endocardial and epicardial levels respectively. Local activation time (LAT) histogram showed incomplete ventricular tachycardia cycle length (70\%) despite best efforts of comprehensive mapping during ventricular arrhythmia.

C \& D figures showing concurrent epi/endocardial electro-anatomical shell with projected radiofrequency ablation lesions from both sides in attempt to bracket the intra-mural myocardial substrates.

Supplemental Video:

Simultaneous epicardial and endocardial activation mapping (CARTO CONFIDENSE ${ }^{\mathrm{TM}}$ Ripple Mapping) during ventricular tachycardia. Activation timing gap is noted during continues activation between the two opposite surfaces with the lack of intra-mural activation mapping.

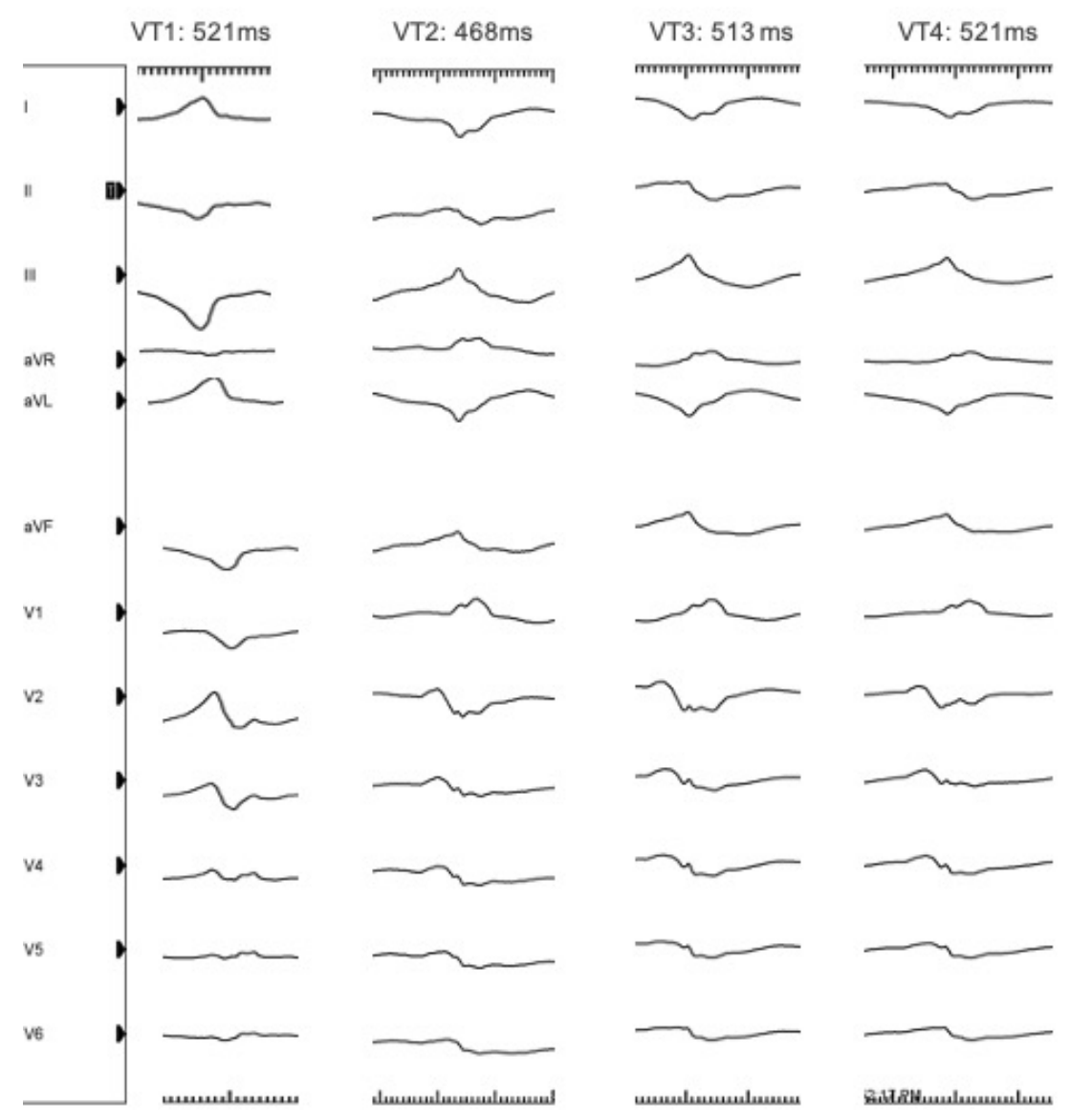



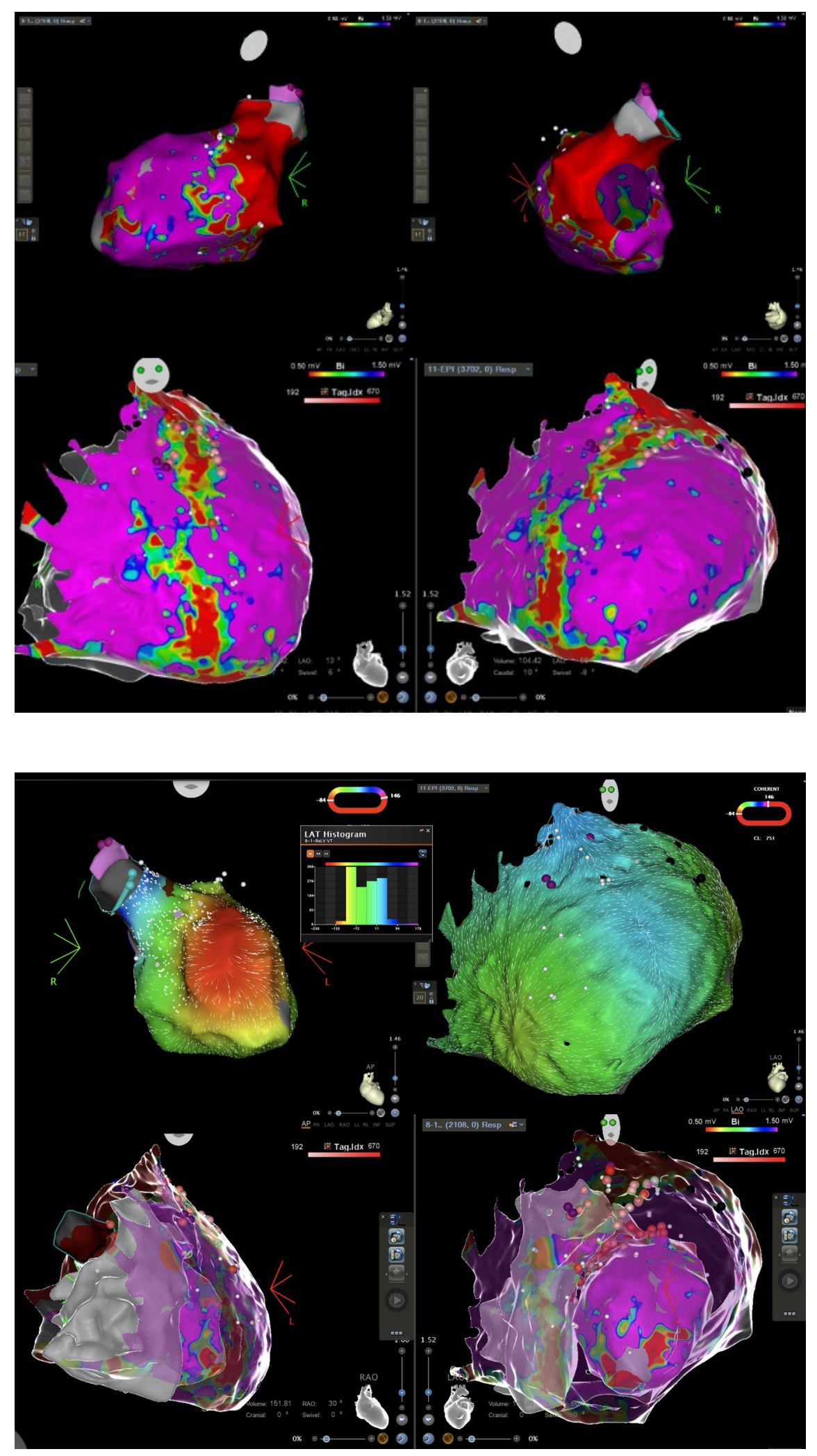